\title{
COMPARACIÓN DE DOS TÉCNICAS DE SALTO CON CAÍDA, PARA MEJORAR EL RENDIMIENTO DE LA POTENCIA DE PIERNAS EN JUGADORES DE BALONCESTO
}

\author{
Jessenia Hernández Elizondo y Walter Salazar \\ Escuela de Educación Física y Deportes \\ Universidad de Costa Rica, San José, Costa Rica
}

\begin{abstract}
Resumen
Hernández Elizondo, J. y Salazar, W. (2001). Comparación de dos técnicas de salto con caída, para mejorar el rendimiento de la potencia de piernas en jugadores de baloncesto. Revista de Ciencias del Ejercicio y la Salud, 1(1), 34-41. El propósito de este estudio fue comparar cuál técnica de salto con caída (piernas flexionadas o extendidas), produce mayores beneficios en la ejecución del salto vertical. Los participantes, $n=34$ de sexo masculino, con una edad promedio de16 años seleccionados de Baloncesto Colegial, con aproximadamente 4 años de experiencia en este deporte, fueron divididos en dos grupos experimentales de ejercicio pliométrico, con un volumen de trabajo de 3 sesiones por semana, durante 8 semanas, realizando en promedio 40 saltos por sesión. El tratamiento consistió en caer desde una grada de $35 \mathrm{~cm}$ y rebotar lo mas alto posible, el grupo experimental 1 que trabajó con las piernas flexionadas $(n=17)$ al caer de la grada, rebotaban llevando las rodillas al pecho $(P F)$, y el grupo experimental $2(n=17)$ que, al caer de la grada, rebotaba con las piernas extendidas. Para determinar el efecto del tratamiento en los sujetos se utilizaron tres tipos de pruebas: la prueba de salto vertical con contramovimiento (SVCC), salto vertical sin impulso (SVSI) y salto largo sin impulso (SLSI). Los datos fueron analizados por medio de una ANOVA de $2 \times 2$ (grupos X mediciones) para cada una de las pruebas realizadas, análisis de efectos simples, omega cuadrado y porcentaje de cambio. Los resultados indican que en todas las pruebas realizadas, ambos grupos experimentales (experimental 1 y 2) tuvieron un cambio en centímetros de aproximadamente $9 \mathrm{~cm}$, $7 \mathrm{~cm}$, y $18 \mathrm{~cm}$, para las pruebas de SVCC, SVSI y SLSI respectivamente, mejoraron significativamente sus resultados entre pre test y post test. En cuanto al análisis de los tratamientos, en la prueba de salto largo SLSI, se presentó una interacción significativa, lo cuál indica que el grupo que trabajó con piernas flexionadas $P F$, mejoró significativamente $(p<0.05)$ su rendimiento. PALABRAS CLAVES: Entrenamiento deportivo, potencia, salto pliométrico.
\end{abstract}

\section{INTRODUCCIÓN}

En una gran cantidad de ocasiones, los deportistas se ven obligados a desarrollar altísimos niveles de fuerza en períodos muy cortos de tiempo. En el mundo de la actividad física encontramos que la masa a desplazar y las velocidades a desarrollar varían considerablemente en función de la modalidad deportiva. El salto vertical es una de las habilidades de mayor importancia en varios deportes.

El caso del Baloncesto no es la excepción ya que en el transcurso del juego se deben hacer múltiples saltos empleando el máximo de potencia para ubicarse en la máxima altura posible, con el fin de conseguir la posición del balón.

Para Díaz y Molina (1994) el entrenamiento pliométrico es un método que posee la capacidad de mejorar, además de la fuerza, la potencia y la capacidad atlética. Esa es la razón por la que para muchos autores los ejercicios pliométricos son el método más utilizado para el desarrollo del poder explosivo de los músculos extensores de las piernas. Este es quizas la forma de entrenamiento más intensa que se pueda practicar, teniendo la ventaja de que es fácil de enseñar y aprender, por lo que es 
actualmente utilizado por un sin número de entrenadores y atletas.

Los ejercicios pliométricos son todos aquellos realizados con el propio peso corporal que impliquen el ciclo estiramientoacortamiento, permitiendo también la actuación de los órganos tendinosos de golgi y husos musculares. (Ortíz, 1996). A su vez este tipo de ejercicio mejora la capacidad para activar un mayor número de fibras musculares en el menor tiempo posible, y da como producto mayor potencia en el atleta. (Peralta, 1993). Bosco (1985), señala que durante la ejecución, dependiendo de la intensidad y tipo de salto, se estimulan muchos cambios a nivel neuromuscular, lo cuál produce cambios rápidos y poderosos a lo largo de los músculos.

Según Weineck (1988), esta técnica de entrenamiento utiliza tres factores dentro del músculo: el reflejo de extensión, la preinervación y los componentes elásticos. En el momento del salto, se produce un aumento de fibras musculares activadas por la acción del reflejo de extensión, durante el contacto con el suelo la preinervación del músculo asume una participación importante por cuanto crea un fondo de inervación óptima para la actividad subsiguiente y modifica el estado de tensión ( elasticidad muscular) que es responsable del tamaño y rapidez de la extensión muscular. Los componentes elásticos del músculo son utilizados como almacenadores de energía cinética, que posteriormente se añade a la contracción muscular concéntrica.

No obstante esa energía sólo puede ser reutilizada si el período de transición entre las fases excéntrica y concéntrica es corto, ya que conforme transcurre el tiempo, la tensión cae y la energía elástica almacenada se pierde. (Miller y Power, 1982). Sin embargo Aragón (1994) afirma que la obtención de una mayor eficiencia en la acción muscular, no necesariamente se debe al almacenamiento de energía elástica, sino que la elasticidad del tendón, podría afectar la potencia mecánica, al permitir que el acercamiento entre el origen y la inserción del músculo tenga una velocidad mayor que la velocidad de acortamiento de las fibras musculares y permitir un rango de movimiento mayor a las articulaciones, teniendo ventaja de esta manera el tendón menos rígido o más elástico.

Vega (1993), Bosco (1994), Miller y Power (1982), Dowling y Vamos (1993) hacen referencia a la explicación de funcionamiento del entrenamiento pliométrico, el cual consiste en que el atleta debe subirse a una plataforma de una altura determinada y dejarse caer para que, al tocar la superficie del suelo, salte inmediatamente, aquí el peso del cuerpo y la gravedad se combinan para crear una resistencia que provoca una fuerte carga en las piernas.

A pesar de la explicación anterior, ningún autor detalla cuál es la colocación de cada uno de los segmentos corporales a la hora de la ejecución de este tipo de saltos, y la mayoría de entrenadores e investigadores asumen, que la técnica que cada uno utiliza es la misma que utilizan los demás, sin embargo esta premisa no necesariamente se da, ya que en la práctica la ejecución es diferente. Delcore (1998), encontró, mejoras en el salto vertical de hasta $12 \mathrm{~cm}$, cuando ejecutaron salto pliométrico llevando las rodillas al pecho después de la caída, pero no comparó con el estilo tradicional, por lo tanto surge esta investigación, con el propósito de determinar cuál técnica de salto con caída, produce mayores ganancias en el rendimiento de la potencia de piernas, fue diseñada esta investigación, la cuál analizará, cuál tratamiento, si el grupo que extiende los brazos y piernas después de la caída o el grupo que lleve las rodillas al pecho, va a obtener mejores resultados en las pruebas de salto vertical y salto horizontal.

\section{METODOLOGÍA}

\section{Sujetos}

La población de este estudio, estuvo compuesta por 34 sujetos varones con edades comprendidas entre 16 y 17 años, los cuales 
son seleccionados de baloncesto colegial, en diferentes colegios del país.

\section{Instrumentos de medición}

La medición de la potencia, se hizo mediante tres pruebas, a saber:

Pruebas de potencia:

Salto vertical con contra movimiento (SVCC), validez: 0.78 , confiabilidad: 0.9. Descripción: El ejecutante se llena con tiza en polvo los dedos corazón e indice, para luego colocarse debajo del saltímetro, (instrumento especialmente diseñado para el salto vertical) colocando la mano que tiene tiza, extendida hacia arriba, con el objetivo de marcar su máximo alcance. Flexionando sus piernas y realizando un balanceo coordinado con los brazos, toma impulso, para tratar de saltar lo más alto posible, marcando con sus dedos el punto más alto que logró tocar. Esta prueba se realiza tres veces, y luego se miden las tres distancias entre el alcance y los tres puntos que realizó, para luego sacar un promedio de los tres resultados.

Salto vertical sin impulso (SVSI). Descripción: Se repite el mismo procedimiento que el SVCM, colocando las manos en la cintura.

Salto Largo sin impulso (SLSI), validez: $\quad 0.60, \quad$ confiabilidad: 0.96 . Descripción: El ejecutante se coloca detrás de la línea de partida que se encuentra marcada en el piso, flexionando sus piernas y realizando un balanceo coordinado con los brazos, toma impulso, para tratar de saltar lo más largo posible., marcando la distancia máxima con su parte del cuerpo que haya quedado más cercana a la línea de salida. Esta prueba se realiza tres veces, y luego de cada intento se miden las tres distancias entre la línea de salida y la máxima distancia alcanzada por el ejecutante, para luego sacar un promedio de los tres resultados.

\section{Procedimientos de medición}

Se definieron al azar, dos grupos experimentales de ejercicio pliométrico, con un volumen de trabajo de tres (3) sesiones por semana durante ocho (8) semanas, realizando la medición de las pruebas al iniciar el tratamiento ( pre-test) y al finalizar el mismo (post-test). Ambos grupos saltaban desde una grada de 35 $\mathrm{cm}$ de alto, siguiendo la siguiente progresión semanal de tratamiento (Delcore,1998):

\begin{tabular}{|c|c|c|c|}
\hline Semana & Series & repeticiones & Recuperación \\
\hline 1 & 3 & 10 & $2 "$ \\
\hline 2 & 3 & 12 & $2 "$ \\
\hline 3 & 4 & 10 & $1 ' 45^{\prime \prime}$ \\
\hline 4 & 4 & 12 & $1 ' 45^{\prime \prime}$ \\
\hline 5 & 4 & 12 & $1 ' 45$ ' \\
\hline 6 & 4 & 14 & 1'30" \\
\hline 7 & 5 & 10 & 1'30" \\
\hline 8 & 5 & 12 & 1'30', \\
\hline
\end{tabular}

El grupo de piernas extendidas (PE), al caer de la grada, rebotaba con las piernas y brazos totalmente extendidos, mientras que el grupo de piernas flexionadas (PF), al caer de la grada, rebotaba llevando las rodillas al pecho.

\section{Análisis estadístico}

Se calcularon los promedios de los intentos para cada sujeto en cada una de las pruebas de salto. Además los datos fueron analizados por medio de un análisis de varianza ANOVA de 2 x 2 (grupos $\mathrm{x}$ mediciones), para cada una de las pruebas realizadas, se realizó un desglose de efectos simples, análisis de omega cuadrado y porcentajes de cambio, para ambos grupos en todas las pruebas.

\section{RESULTADOS}

La estadística descriptiva (promedio y desviación estándar) de cada grupo se presenta en la tabla \#1.

La tabla \# 2 muestra la estadística inferencial (anova y Omega Cuadrado) para cada una de las pruebas de salto vertical, los datos que tienen asterisco $(*)$ son los que 
presentaron una diferencia significativa para $\mathrm{p}<0.05$.

Los resultados que muestra la tabla \# 2, nos indican que hubo un cambio significativo, en la variable B de mediciones (pre test y post test). Sin embargo vemos que la interacción no fue significativa, por lo tanto se deduce que ambos tratamientos, (grupo PE y grupo PF), mostraron una mejoría de igual magnitud.

Opuesto a lo anterior, tenemos en la tabla \# 3, que para la prueba de salto largo sin impulso (SLSI) se dio una interacción significativa (ver gráfico \# 1), lo cuál nos indica uno de los tratamientos, provocó un cambio mayor que el otro, en este caso el grupo PF mejoró significativamente más que el grupo PE.

De acuerdo con la tabla \# 2, es importante conocer en cuál de los dos grupos, mostró un cambio mayor. Así la tabla \# 3 indica que para la prueba de SLSI, el grupo que trabajó llevando las rodillas al pecho, inmediatamente después de la caída $(\mathrm{PF})$, mejoró significativamente con respecto al grupo que trabajó con las piernas extendidas (PE).

Tabla\#1

Estadística descriptiva para ambos grupos experimentales.

\begin{tabular}{lcccc}
\hline & \multicolumn{2}{c}{ Piernas Flexionadas } & \multicolumn{2}{c}{ Piernas Extendidas } \\
\cline { 2 - 5 } & pre & Post & pre & post \\
\cline { 2 - 5 } SVCC & $48.47 \pm 8.58$ & $56.35 \pm 9.16$ & $47.86 \pm 6.97$ & $55.80 \pm 7.21$ \\
SVSI & $48.29 \pm 8.04$ & $55.22 \pm 7.97$ & $43.82 \pm 8.06$ & $50.78 \pm 8.38$ \\
SLSI & $2.03 \pm 15.46$ & $2.24 \pm 19.21$ & $1.93 \pm 15.96$ & $2.07 \pm 18.35$ \\
\hline
\end{tabular}

El gráfico \# 2, muestra que el grupo de piernas flexionadas, obtuvo un porcentaje de cambio de un $16,25 \%$ para la prueba de SVCC, un $14,35 \%$ para el SVSI y un $10,34 \%$ para el SLSI. Por su parte el grupo de piernas extendidas, mejoró en un $17,92 \%$, $15,88 \%$ y $7,25 \%$ para las pruebas de SVCC, SVSI y SLSI respectivamente.

Tabla \#2

Resumen de ANOVAS para las pruebas de salto vertical con contramovimiento SVCC y salto vertical sin impulso SVSI.

\begin{tabular}{clcc}
\hline Prueba & Fuente de varianza & $\mathbf{F}$ & $\boldsymbol{\omega}^{\mathbf{2}} \mathbf{( \% )}$ \\
\hline \multirow{3}{*}{ SVSI } & Tratamientos(A) & 2.60 & 4 \\
& Mediciones (B) & $421.23^{*}$ & 15 \\
& A*B & 0.003 & 0 \\
\hline SVCC & Tratamientos(A) & 0.045 & 0 \\
& Mediciones (B) & $304.98^{*}$ & 20 \\
& A*B & 0.004 & 0 \\
\hline
\end{tabular}

$* \overline{\mathrm{F}(1,32) 0.05: 4.15}$

A pesar de que el porcentaje de cambio para la prueba de salto largo sin impulso fue menor, esto se debe a la proporción de mejoría en centímetros, con respecto a la totalidad de $\mathrm{cm}$ alcanzados en la prueba. Por esta razón es importante señalar la magnitud del cambio en $\mathrm{cm}$ entre pre test y post test para cada una de las pruebas, que 
se muestra en el gráfico \# 3, donde para el SVCC, el grupo de PE mejoró en promedio $8 \mathrm{~cm}$, mientras que en el grupo PF el aumento fue de $10 \mathrm{~cm}$. Por su parte ambos grupos PE y PF mejoraron $7 \mathrm{~cm}$ en la prueba de SVSI, por último en la prueba de SLSI, el grupo PE aumentó en $14 \mathrm{~cm}$, mientras que el grupo PF, logró aumentar su salto en $21 \mathrm{~cm}$.

Tabla \# 3

Resumen de ANOVA para la prueba de salto largo.

\begin{tabular}{clcc}
\hline Prueba & Fuente de varianza & F & $\boldsymbol{\omega}^{\mathbf{2}}(\boldsymbol{\%})$ \\
\hline \multirow{3}{*}{ SLSI } & Tratamientos(A) & $4.532^{*}$ & 8 \\
& Mediciones (B) & $297.381^{*}$ & 16 \\
& $\mathrm{~A} * \mathrm{~B}$ & $9.897^{*}$ & 1 \\
\hline
\end{tabular}

$* \overline{\mathrm{F}(1,32) 0.05: 4.15}$

\section{DISCUSIÓN}

Este estudio fue diseñado con el propósito de comprobar cuál era la técnica de salto con caída (piernas extendidas PE y piernas flexionadas $\mathrm{PF}$ ) que produce mayores beneficios en la ejecución del salto vertical y horizontal, en un grupo de jugadores de baloncesto de categoría juvenil.

En cuanto al rendimiento de los sujetos en cada una de las tres pruebas (SVCC, SVSI y SLSI), se concluye que entre pre test $\mathrm{y}$ post test, hubo un cambio significativo en ambos grupos experimentales (PF y PE). En la prueba de salto largo sin impulso (SLSI), el grupo que trabajó llevando las rodillas al pecho inmediatamente después de la caída PF, obtuvo resultados significativamente mayores que el grupo que trabajó con las piernas extendidas (PE) lo cuál tiene relación con Díaz \& Molina (1994) quienes encontraron, después de comparar dos grupos, uno que entrenó solamente ejercicios pliométricos y otro que trabajó con pesas, que el grupo de pliométricos obtuvo mayor rendimiento en el salto largo, mientras que el grupo que entrenó con pesas mejoró significativamente las pruebas de salto vertical. Por otro lado Piedra (1995) luego de manipular alturas en el salto pliométrico, encuentra resultados disímiles, cuando señala una mejoría significativa en las pruebas de salto vertical, con respecto al salto horizontal.

No obstante, cabe recalcar que las magnitudes de cambio para cada grupo experimental (piernas extendidas PE y piernas flexionadas $\mathrm{PF}$ ) en las pruebas de salto vertical, a pesar de no ser estadísticamente significativas, si lo son en la práctica real, ya que el aumento de $7 \mathrm{~cm}$ para el SVSI y $10 \mathrm{~cm}$ en el $\mathrm{SVCC}$, podrían representar para los entrenadores grandes ganancias en el rendimiento de sus atletas

Tabla \# 3.a.

Desglose de efectos simples para la prueba de salto largo.

\begin{tabular}{lcc}
\hline \multicolumn{1}{c}{ Fuente de varianza } & F & $\boldsymbol{\omega}^{\mathbf{2}}(\boldsymbol{\%})$ \\
\hline A 1 (flexionadas) PF & $5.14^{*}$ & 9 \\
A 2 (extendidas) PE & 2.43 & 3 \\
\hline
\end{tabular}

$* \mathrm{~F}(1,32) 0.05: 4.15$ 


\section{Gráfico \# 1}

Interacción entre el estado de las piernas y las mediciones en el salto largo.

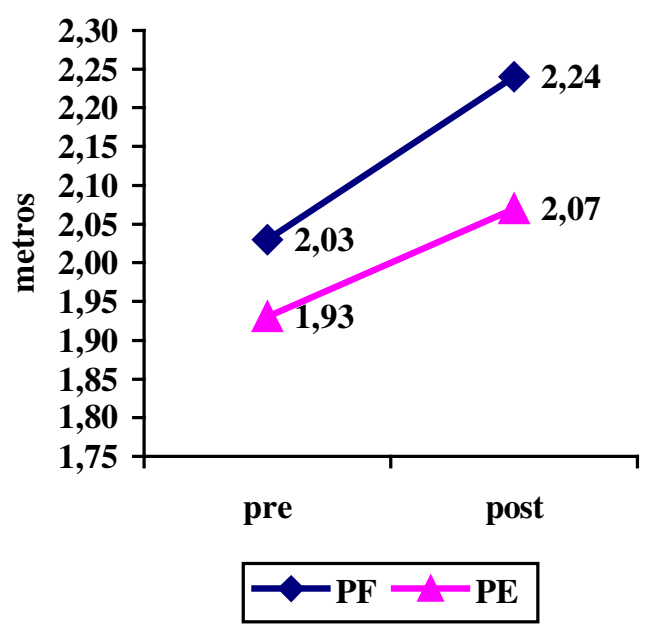

Una de las razones por las que un entrenamiento de tipo vertical, tuviera efectos positivos en una prueba horizontal (SLSI) podría estar fundamentada en el principio de transferencia, el cual menciona, que la condición para que ésta se dé, es que existan coincidencias coordinativas entre los movimientos respectivos. Berstein (1967) señaló que lo importante para que se dé la transferencia es la coincidencia entre los mecanismos coordinativos sensomotores y no las semejanzas externas en el desarrollo del movimiento.

Gráfico \# 2

Porcentaje de cambio entre el pre test y post test en cada una de las pruebas realizadas

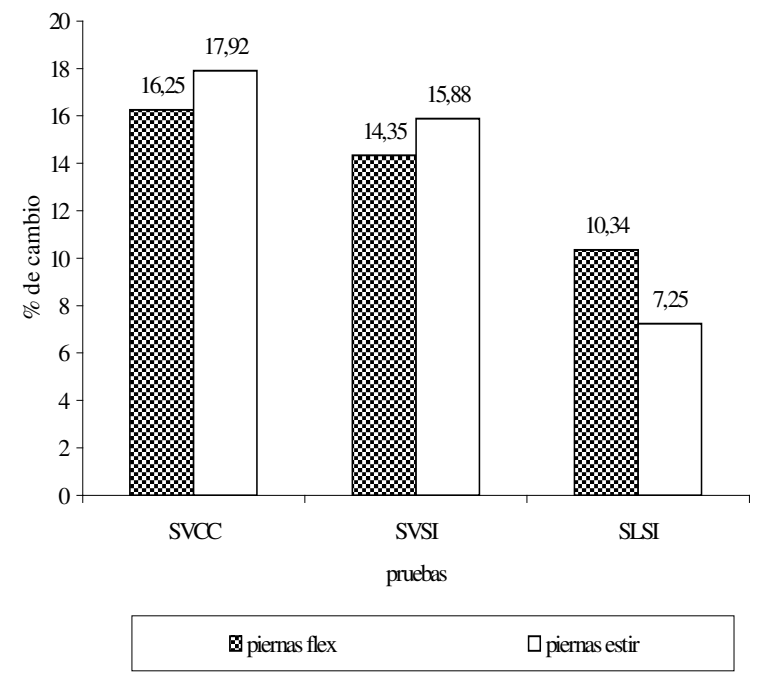


Por otro lado, dos teorías pueden explicar, el hecho de que para las pruebas de salto vertical con contra movimiento (SVCC) y salto vertical sin impulso (SVSI), no se hayan presentado diferencias entre las mejorías. Primero: que al existir una influencia por un factor de aprendizaje, los resultados del grupo PF tuvieran mejores resultados para el salto largo, ya que existe un movimiento similar de recoger las piernas, elevando las rodillas durante el vuelo, en ambas situaciones. Segundo: $\mathrm{Si}$ observamos el gráfico \# 2, el grupo de piernas extendidas obtuvo un mayor porcentaje de cambio en las pruebas de salto vertical, donde ambas situaciones son similares, cuando los sujetos llevan los brazos y piernas totalmente extendidas en la fase de vuelo.

Bosco (1994) afirma que en pruebas de la valoración de la fuerza explosiva, como el test de sargent, es imposible separar la influencia que ejerce la coordinación de los miembros superiores y su contribución en la obtención de energía elástica, la cuál puede afectar el componente contractil de los músculos extensores de las piernas. Este planteamiento concuerda con Dowling y Vamos (1993) quienes afirman que el balanceo de brazos mejora la altura del salto.

Gráfico \# 3

Magnitud de cambio en (cm) entre pre test y post test en cada una de las pruebas.

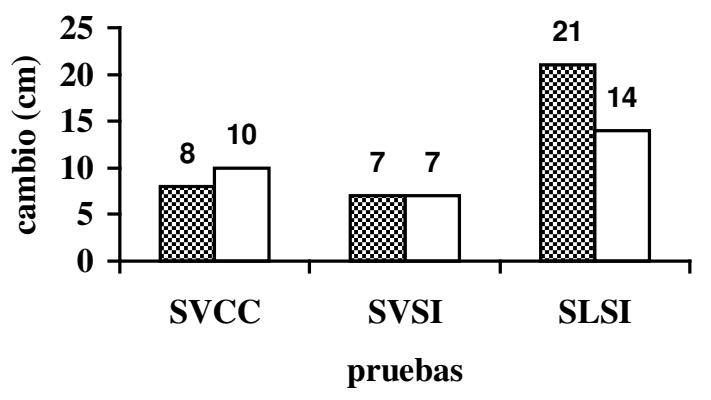

MPF $\square$ PE

No obstante, la literatura consultada tiene como objetivo principal comparar los resultados obtenidos antes y después de un tratamiento determinado. Para Aragón (1996) lo más importante es que en el transcurso de toda la investigación se utilice el mismo método o prueba y su validez sea buena.

Resumiendo los principales hallazgos encontrados en esta investigación, se recomienda el entrenamiento de ejercicios pliométricos con elevación de rodillas al pecho, inmediatamente después de la caída, cuando se quiera mejorar el rendimiento en el salto horizontal. Sin embargo ambos tipos de tratamiento (grupo de PE y grupo de PF) mejoran el salto vertical en más de $7 \mathrm{~cm}$, lo cual representa una gran ganancia en el rendimiento de los deportistas.

\section{REFERENCIAS}

Aragón L F (1994) Papel de la elasticidad del tendón en la potencia muscular. Memoria del I Congreso Internacional sobre entrenamiento deportivo. Editado por Universidad de Costa Rica. Aragón L F (1996) Comparación de cuatro métodos para el salto vertical. Revista Educación, (en prensa)

Bosco, C (1985) Stretch-Shortening cycle in skeletal muscle function, and physiological consideration on explosive power in men. Atleticastudi,1: 67-113 
Bosco, C (1994) La valoración del test de Bosco. Editorial Paidotribo.

Delcore,G (1998) Comparación en la mejoría del salto vertical en jugadoras de volibol, entre el entrenamiento pliométrico con una pierna y dos piernas. Escuela de Educación Física y Deportes. Universidad de Costa Rica.

Díaz, J \& Molina, R (1994) Desarrollo de la fuerza, velocidad y potencia en los músculos extensores de la pierna: una comparación entre el entrenamiento pliométrico y pesas. Tesis. Escuela de Educación Física y Deportes. Universidad de Costa Rica.

Dowling, J \& Vamos, L (1993) Identification of kinetic and temporal factors related to vertical jump performance. Journal of Applied Biomechanics, 9: 95-110

Johnson B \& Nelson J. (1974) Practical measurements for evaluation in Physical Education. $\mathbf{2}^{\mathbf{a}}$ Ed. Minneapolis, Minesota U.S.A: Burgess Publishing.

Miller B \& Power, S (1982) El salto en profundidad como medio de desarrollo de la potencia en atletismo. Cuaderno de Atletismo. Acondicionamiento físico deportivo. Real Federación Española de Atletismo.

Ortíz, C V (1996) Entrenamiento de fuerza y explosividad para la actividad física y el deporte de competición. $\mathbf{1}^{\text {a }}$ Ed. INDE publicaciones. Barcelona España.

Peralta, H. (1993)Hacia las posibilidades infinitas de la enseñanza de la gimnasia. Editorial C.I.P. Bogotá. Colombia.

Piedra, D (1995) Entrenamiento de ejercicio pliométrico basados en la altura óptima de salto y su influencia en la fuerza, velocidad y potencia. Tesis.

Escuela de Educación Física y deportes.

Universidad de Costa Rica.

Vega, J (1993) Efecto de dos tipos de entrenamiento pliométrico en el mejoramiento de la habilifdad del salto vertical en jugadoras de fútbol universitarias. Memoria XIV Congreso Panamericano de Educación Física, vol. 1,79-86.

Weineck, Jürgen. (1988) Entrenamiento

óptimo. Editorial Hispano Europea. Barcelona España. 